\title{
Plataforma Microsoft Teams y su influencia en el aprendizaje de estudiantes de básica superior
}

\section{Microsoft Teams platform and its influence on the learning of upper basic students}

\author{
Cecilia Maricela Rodríguez-Guijarro \\ cecilia.rodriguez.64@est.ucacue.edu.ec \\ Universidad Católica de Cuenca, Azogues \\ Ecuador \\ https://orcid.org/0000-0003-0835-2783 \\ Ana Zulema Castro-Salazar \\ azcastros@ucacue.edu.ec \\ Universidad Católica de Cuenca, Azogues \\ Ecuador \\ https://orcid.org/0000-0002-3837-314X
}

Recepción: 10 de abril 2021

Revisado: 05 de mayo 2021

Aprobación: 30 de junio 2021

Publicación: 15 de julio 2021 


\title{
RESUMEN
}

El objetivo de la investigación se basa en analizar el uso de la plataforma Microsoft Teams y su influencia en el aprendizaje de estudiantes de Básica superior de la unidad educativa "16 de abril "de la ciudad de Azogues. Metodológicamente fue de tipo descriptiva con diseño no experimental. Entre los resultados, se encontró que la plataforma Teams es de gran utilidad, según los resultados obtenidos se puede notar que los estudiantes y las docentes se sienten cómodos con la aplicación que utilizan ya que posee carteristas que otras plataformas no tienen, de igual e incluso podemos hacer una lección oral creando formularios interactivos.

Descriptores: Pandemia; resiliencia; proceso de comunicación; aprendizaje activo. (Palabras tomadas del Tesauro UNESCO).

\begin{abstract}
The objective of the research is based on analyzing the use of the Microsoft Teams platform and its influence on the learning of Upper Basic students of the educational unit "April 16" in the city of Azogues. Methodologically, it was descriptive with a nonexperimental design. Among the results, it was found that the Teams platform is very useful, according to the results obtained, it can be noted that students and teachers feel comfortable with the application they use since it has pickpockets that other platforms do not have, equally and even we can do an oral lesson creating interactive forms.
\end{abstract}

Descriptors: Pandemics; resilience; communication process; activity learning. (Words taken from the UNESCO Thesaurus). 


\section{INTRODUCCIÓN}

A nivel mundial, la educación ha tomado un giro de lo presencial a lo virtual, debido a, la pandemia del COVID 19. A causa de esto, el mundo se sometio a un encierro forzoso, que ha afectado severamente la vida cotidiana de hombres, mujeres y niños. Este aislamiento hizo que las actividades: educativas, sociales y económicas, entre otras, se paralizaran. Todo esto dio paso al uso de diferentes plataformas de comunicación y gestión de información en todos los campos, especialmente en la educación, lo cual, provocó un nuevo impulso en el modelo de educación a distancia virtual.

En cuanto a Ecuador, el ministerio de educacion de la replublica del Ecuador solicito el uso de la plataforma digital Microsoft Teams en el proceso de enseñanza aprendizaje, debido al confinamiento por covid-19, este seria el medio de interaccion entre docentes y estudiantes, por otro lado el aprendizaje podra ser mas dinamico utilizando tecnicas y estrategias que fometen saberes en realcion a la naturaleza y todos los que habitan en el planeta tierra (Mendoza-Noriega et al. 2020).

Sin embargo, desde que incio el lectivo 2020-2021 en el sector sierra existe gran desmotivacion para hacer uso del mismo, esto debido reportes negativos para ingresar y falta de capacitacion e el manejo, lo que a dessencadenado, que los docentes utilicen otras alternativas como: Classroom, Neo Lms, Whatsapp y muy pocas utilizan Microsoft Teams con el fin,de acompañar en el proceso pedagogico virtual.

En este contexto, (Mendoza-Noriega et al. 2020), mencionan que sin educacion los pueblos fracasan, por lo que el estado Ecuatoriano de garantizar una educacion de calidad para todos, por lo que es de suma importacia que las entidades minesteriales pongan mas interes en los actores educativos para evitar desfaces en la cimentacion de conocimientos.

Por lo cual, el objetivo de la investigación se basa en analizar el uso de la plataforma Microsoft Teams y su influencia en el aprendizaje de estudiantes de Básica superior de la unidad educativa "16 de abril "de la ciudad de Azogues- Ecuador. 


\section{Referencial teórico}

Se presenta a (Vargas-Cubero \& Villalobos-Torres, 2017), quienes realizaron una investigación titulada "El uso de plataformas virtuales y su impacto en el proceso de aprendizaje en las asignaturas de las carreras de Criminología y Ciencias Policiales, de la Universidad Estatal a Distancia de Costa Rica", la cual, tenía como objetivo determinar cómo incide el uso de una plataforma educativa online en el aprendizaje de los estudiantes las carreras de Ciencias Criminológicas y Ciencias Policiales.

Ahora bien, esta investigación obtuvo como resultados que el uso de plataformas y de tecnología contribuyen a potenciar y favorecer el aprendizaje, no obstante, también se comprendió que la tecnología y la plataforma educativa por sí solas no marcan la diferencia, por lo tanto, se requiere organización de las asignaturas, apoyos y principalmente la mediación de los docentes para que el aprendizaje sea significativo.

Mientras que (Sánchez-Palacios, 2020), realizó una investigación titulada "Impacto del Aula Virtual en el Proceso de Aprendizaje de los Estudiantes de Bachillerato General", la cual, tenía como objetivo analizar las cualidades positivas que brinda un aula virtual en el aprendizaje de los estudiantes del bachillerato general. Por lo que, esta investigación obtuvo como resultado, que el estudiante con el uso del aula virtual podrá construir su propio conocimiento, para lo cual, es fundamental tener conocimientos básicos en aplicaciones informáticas y tecnología, además ayuda a que el docente desempeñe nuevas estrategias para la enseñanza.

En cuanto a (Rivera-Villalta et al. 2020), en su artículo titulado "Formación de competencias tecnológicas en el uso de Microsoft Teams en los estudiantes del bachillerato", en la cual aplicaron Microsoft Teams como plataforma educativa. Obteniendo como resultado que las herramientas TIC como medio de transmisión y generación de conocimientos se pueden convertir en muy eficaces, además, por medio de la encuesta se pudo analizar la viabilidad de usar un software de enseñanzaaprendizaje, teniendo en cuenta que ese rol fundamental y el de comunicación entre 
docente y alumno no puede ser remplazado por un software o una máquina, pero es mejor hacer uso de un software educativo que por redes sociales.

\section{Plataformas virtuales educativas}

Desde tiempo atrás, la tecnología ha influenciado en el ámbito educativo, dentro del proceso enseñanza-aprendizaje, facilitando los roles del docente como también del estudiante, misma que abre un campo que dicho proceso que no sea solo en una, aula física. Es por ello que ha brindado una gama de plataformas virtuales, donde el docente es un mediador o facilitador y el alumno el constructor de su conocimiento siendo motivado constantemente.

Las plataformas virtuales, son sistemas que nos permiten ejecutar varias aplicaciones en un mismo entorno, por lo que da a los usuarios la posibilidad de acceder a través de Internet, además, se utilizan para la educación a distancia y pretenden simular los mismos escenarios de aprendizaje que se registran en un aula; Es decir una plataforma virtual educativa, es un programa que abarca varias herramientas destinadas a la organización de información, asignación de tareas, interacción entre el docente y estudiantes.

El conjunto de aplicaciones informáticas de tipo asincrónico y sincrónico es una plataforma virtual, que atreves de la internet facilita el desarrollo, la gestión y distribución de cursos. Para proveer este servicio a la comunidad, el software debe ser instalado en el servidor de la institución; Es decir, es un sistema que abarca varias herramientas destinadas a la organización de información, asignación de tareas, interacción entre el docente y estudiantes, simulando los mismos escenarios de aprendizaje que se registran en un aula, por lo que es muy utilizada en la educación a distancia. (Pando, 2018).

Al respecto (Sánchez-Rodríguez, 2009), manifiestan que las plataformas virtuales contienen una serie de características y herramientas entre ellas la comunicación de forma directa e indirecta, la administración siendo factible la orientación y apoyo al estudiante. Por ello, la situación mundial en la que vivimos, covid-19, vemos dichos recursos como indispensables debido a que podemos trabajar de forma sincrónica con 
los estudiantes que poseen internet fijo, y también de manera asincrónica con quienes poseen internet a través de datos, aprovechando lo que ofrece cada una de ellas.

Por otra parte, existe gran variedad de plataformas educativas y la mayoría coinciden en mostrar a los usuarios las múltiples funciones como: crea y gestionar diferentes cursos en la web, sin que sean necesarios tener conocimientos avanzados de programación y diseño gráfico. Esto se debe a que en cada plataforma vienen incluidas las plantillas prediseñadas, mismas que día a día van mejorando por la presión del avancé tecnológico. En este contexto las plataformas virtuales pueden suelen agruparse en: plataformas comerciales, plataformas de software libre y plataformas de desarrollo propio de las instituciones.

De acuerdo a (Lagunes-Domínguez \& Lagunes-Domínguez, 2018), cada una de ellas se pueden definir de la siguiente manera: las plataformas de desarrollo propio, estas son desarrolladas en la institución acorde a las necesidades y requerimientos propios de la comunidad educativa; las plataformas de software libre no tienen un costo, por lo tanto, no tienen capacitaciones y un soporte para los usuarios; al contrario, las plataformas comerciales tienen un costo, para ser utilizados y otorga ciertos derechos para recibir asesoría técnica, capacitación. Existen un sinnúmero de plataformas comerciales, entre ellas están, Neo LMS, FirstClass, WebCt y Microsoft Teams, entre otras. Cabe recalcar que sin importar su tipo tiene compatibilidad con SCORM, Tin Can API.

\section{Microsoft Teams}

De la generosidad que ofrece la tecnología, en los últimos años se ha utilizado la plataforma Teams de Microsoft, aunque en su inicio tuvo un propósito colaborativo para empresas, su auge llegó al campo educativo. Permitiendo crear un entorno amigable y motivador del proceso de enseñanza- aprendizaje, a través de grupos de trabajo donde es posible la intercomunicación de forma instantánea, tales como video llamadas, que pueden grabarse y ser publicadas, además ha incorporado un paquete de office 365 , permitiendo al usuario editar, visualizar, en línea. 
Por otra parte, (Haro-Calero \& Yépez-Pullopaxi, 2020), manifiestan que Microsoft Teams brinda opciones para que el docente y estudiante tengan accesibilidad desde cualquier lugar, es decir desde el sitio web oficial, la versión de casa o de escritorio, y por último la móvil. Sin duda alguna la plataforma Microsoft Teams, en el campo educativo, es una de las más completas, puesto que la facilidad de video llamadas, emitir contenidos, asignar trabajos, publicar calificaciones y la comunicación sincrónica entre docentes y estudiantes.

\section{Microsoft Teams en el aprendizaje}

Por consiguiente, todo lo mencionado genera una notable diferencia entre otras plataformas y Microsoft Teams, debido a que ha incorporado varias funciones en una sola, siendo de gran importancia en el aprendizaje, ya que también existen aplicaciones que cumplen con la evaluación y retroalimentación, además, permite crear salas colaborativas desarrollando las destrezas y el docente ahorra tiempo compartiendo documentos y tareas invirtiendo en la planificación de su clase. En este sentido la plataforma Microsoft Teams es una estrategia metodológica, de fácil acceso solo necesita un usuario y contraseña para su manejo a través de iconos que despiertan el interés en el estudiante, explorando y a su vez encontrando los contenidos y tareas todo ello incentiva el aprendizaje.

También, (Llesquen-Chinga, 2020), indica que Microsoft Teams, brinda la facilidad para generar metodologías innovadoras dentro del proceso de enseñanza-aprendizaje, porque contiene aplicativos donde el estudiante interactúa de forma constante, además permite crear salas colaborativas desarrollando las destrezas, además el docente ahorra tiempo compartiendo documentos y tareas invirtiendo en la planificación de su clase.

En este sentido la plataforma Teams es una estrategia metodológica, de fácil acceso solo necesita un usuario y contraseña para su manejo a través de iconos que despiertan el interés en el estudiante, explorando y a su vez encontrando los contenidos y tareas todo ello incentiva el aprendizaje. Por consiguiente, la plataforma Teams, es de fácil 
accesibilidad para los estudiantes ya que se puede instalar en cualquier dispositivo electrónico que poseen, siendo ideal en el aprendizaje puesto que permite compartir información e invertir roles generando un verdadero conocimiento.

En la misma línea (Hernández et al. 2018), manifiestan que las plataformas virtuales se han vuelto indispensables tanto en la enseñanza como en el aprendizaje, debido que se ha cambiado la forma como el estudiante aprende, de manera constructiva rompiendo las barreras del tradicionalismo, siendo un actor propio del desarrollo de sus conocimientos y habilidades, sin dejar a un lado el guía o facilitador y por ello el docente es quien deberá manejar de forma correcta para que sea él quien permita el desarrollo del estudiantes en sus áreas. Por lo tanto, la plataforma Teams es un aula sin paredes, pero a la vez posee un espacio siendo menester hacer buen uso de los avances tecnológicos y sus herramientas de forma correcta generando un verdadero aprendizaje en diferentes áreas.

\section{METODOLOGÍA}

En esta investigación se empleó el enfoque mixto con una visión descriptiva no experimental de los hechos. Para analizar la población desde una perspectiva cuantitativa, se empleó una muestra estratificada, en este tipo la muestra permite la selección de un grupo específico y se convertiría en una subpoblación de una población. Por lo tanto, la muestra está compuesta por 58 estudiantes del noveno año de EGB, con un nivel de confianza del $99 \%$ y con un margen de error del $1 \%$, y 2 docentes de la unidad educativa donde se realiza la investigación.

En este contexto, el instrumento encuesta fue aplicado a los estudiantes y después de realizar el análisis en SPSS, la variable obedece a que todas son paramétricas según las pruebas de normalidad Kolmogorov y el Alfa de Cronbach es de 0.708 , por otro lado, a las docentes se les aplico la entrevista que fue validada por el método Delphi.

En este análisis en SPSS se determinó los estadísticos descriptivos y la tabla de frecuencias para arribar a conclusiones y establecer la influencia de la plataforma 
Microsoft Teams en el aprendizaje de los estudiantes de la unidad educativa "16 de abril "de noveno año de Educación General Básica. Para la fase cualitativa, se trabajó con entrevista abierta y codificación de las cualidades aportadas por los informantes clave.

\section{RESULTADOS}

Los resultados obtenidos al aplicar los intrumentos de investigacion se detallaran acontinuacion.

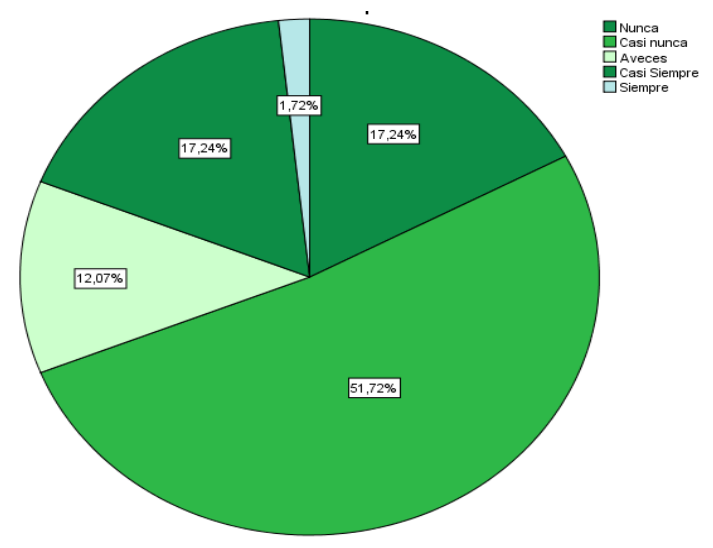

Figura 1. Los estudiantes tienen difiultad al usar la plataforma microsoft teams. Fuente: Encuesta.

La figura 1 muestra que el $51,7 \%$ de los encuestados manifiesta que casi nunca tiene dificultades al utilizar la plataforma Microsoft Teams, por lo que demuestra que los estudiantes has desarrollado competencias tecnológicas lo cual es de vital importancia para potenciar el aprendizaje en la población estudiantil. Ahora bien, es de suma importancia que los docentes estén formados en competencias ya que es un factor determinante para una correcta integración de la plataforma Microsoft Teams en el quehacer académico. 
Revista Arbitrada Interdisciplinaria KOINONIA

Año VI. Vol VI. N³. Edición Especial: Educación II. 2021

Hecho el depósito de Ley: FA2016000010 ISSN: 2542-3088

FUNDACIÓN KOINONIA (F.K). Santa Ana de Coro. Venezuela.

Cecilia Maricela Rodríguez-Guijarro; Ana Zulema Castro-Salazar

\section{Tabla 2.}

Relación entre docentes utilizan estrategias de aprendizaje y Uso de la plataforma para acompañamientos pedagógicos.

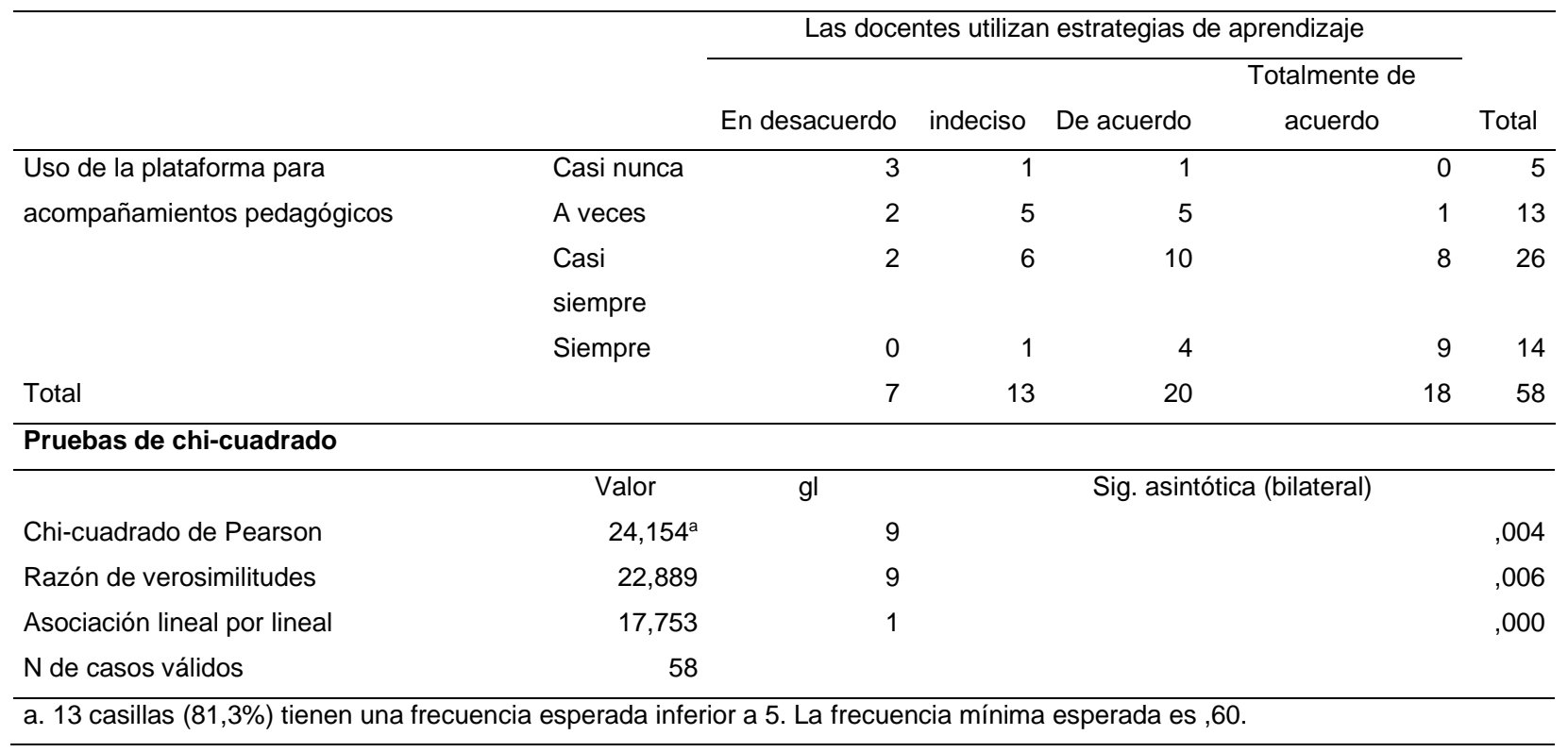

Fuente: Encuesta.

La tabla 2 muestra la relación mediante la prueba chi-cuadrado con un valor de Sig. 004 por lo que es afirmativa, se observa que la población confirma que las docentes usan estrategias como trabajos grupales, organizadores gráficos, resúmenes entre otros, durante los acompañamientos pedagógicos utilizando la plataforma Microsoft Teams, esto incide de manera positiva para que los estudiantes puedan potencia su aprendizaje. 
Revista Arbitrada Interdisciplinaria KOINONIA

Año VI. Vol VI. N³. Edición Especial: Educación II. 2021

Hecho el depósito de Ley: FA2016000010 ISSN: 2542-3088

FUNDACIÓN KOINONIA (F.K). Santa Ana de Coro. Venezuela.

Cecilia Maricela Rodríguez-Guijarro; Ana Zulema Castro-Salazar

\section{Tabla 3.}

Microsoft Teams facilita la enseñanza.

\begin{tabular}{llrrrr}
\hline & Frecuencia & Porcentaje & Porcentaje válido & \multicolumn{2}{c}{$\begin{array}{c}\text { Porcentaje } \\
\text { acumulado }\end{array}$} \\
\hline Válidos & Totalmente de acuerdo & 21 & 36,2 & 36,2 & 36,2 \\
& De acuerdo & 28 & 48,3 & 48,3 & 84,5 \\
& Indeciso & 7 & 12,1 & 12,1 & 96,6 \\
& Totalmente en desacuerdo & 2 & 3,4 & 3,4 & 100,0 \\
& Total & 58 & 100,0 & 100,0 & \\
\hline
\end{tabular}

Fuente: Encuesta.

En la tabla 3 se observa que la población estudiantil encuestada manifiesta que la plataforma Microsoft Teams facilita la transmisión de conocimientos, por lo cual se puede corroborar que usar la plataforma con las estrategias y herramientas adecuadas incide en el aprendizaje de los estudiantes.

Ahora bien, a las docentes se les aplico el instrumento de la entrevista con el objetivo de analizar el uso de la plataforma Microsoft Teams y determinar la influencia en los estudiantes.

\section{Tabla 4.}

Análisis Cualitativo.

\begin{tabular}{llr}
\hline Unidad de Análisis & Categoría & Segmento \\
\hline $\begin{array}{l}\text { Tecnología y } \\
\text { educación }\end{array}$ & $\begin{array}{l}\text { Uso de la } \\
\text { plataforma } \\
\text { Microsoft Teams }\end{array}$ & $\begin{array}{l}\text { La plataforma Microsoft Teams es considerada de } \\
\text { fácil uso debido a que su interfaz es amigable, ya que } \\
\text { contiene múltiples herramientas que ayudan en el } \\
\text { desarrollo de capacidades en los estudiantes. }\end{array}$ \\
& $-\quad \begin{array}{l}\text { Microsoft Teams es una herramienta que se puede } \\
\text { utilizar en diferentes dispositivos tales como Laptop, } \\
\text { celulares, siendo esta también gratuita y de libre uso. }\end{array}$ \\
\hline Estrategias & La plataforma como estrategia metodológica es flexible \\
metodológicas & $\begin{array}{l}\text { porque los estudiantes pueden acceder a las actividades } \\
\text { asignadas a cualquier hora del día para su análisis y y } \\
\text { permita fortaleces sus capacidades. }\end{array}$ \\
\hline
\end{tabular}


- La plataforma permite un aprendizaje individual y colaborativo de modo dinámico y versátil. Por lo que mejora la predisposición del alumnado en el aprendizaje diario

\begin{tabular}{lll}
\hline Experiencia & Influencia de & La plataforma facilita el intercambio de documentos y \\
Teams & contenidos en múltiples formatos como JPG, PNG, \\
& PDW, entre otros. Además, permite la incrustación y \\
& vinculación de diferentes herramientas externas \\
& como: Edpuzzle, Mentimeter, padlet, entre otros. \\
& - Microsoft Teams proporciona diversos recursos \\
& orientados para trabajar de forma personalizada en \\
& los encuentros sincrónicos y asincrónicos.
\end{tabular}

Fuente: Entrevistas.

\section{Unidades de análisis y categorías}

\section{Uso de la plataforma Microsoft Teams}

Microsoft Teams fue otorgada por el ministerio de educación, tiene diversidad de recurso, entre ellos está el paquete de Office 365, se puede utilizar en un navegador o ser instalada en un dispositivo electrónico como: celular, Tablet, Laptop, por lo que, es de fácil instalación y ejecución en los diversos equipos digitales. De acuerdo con las docentes entrevistadas, luego de una auto capacitación en donde desarrollaron habilidades por lo que sostienen que la plataforma es fácil de utilizar, además que permiten administrar tareas, actividades asignadas a los estudiantes por medio de herramientas incluidas en la misma.

\section{Estrategias metodológicas}

El uso de la plataforma Microsoft Teams se ha ido consolidando con el proceso aprendizaje debido a su interfaz capta la atención del estudiante por poseer diversas herramientas de trabajo que ayuda a los estudiantes en el desenvolvimiento de habilidades y capacidades, Ahora bien, las docentes entrevistas indican que en el proceso de enseñanza-aprendizaje utilizan las herramientas para realizar trabajo 
colaborativo, diálogos, entre otras. Es decir, aplican metodologías activas conquistando la predisposición del estudiante en el aprendizaje diario. Por lo que han logrado resultados positivos en el aprendizaje.

\section{Influencia de Microsoft Teams}

En la actualidad la tecnología ha impuesto nuevos hábitos de estudio, en las cuales los docentes deben buscar formas para personalizar la enseñanza-aprendizaje, en este contexto las docentes entrevistadas, dan a conocer en base a su experiencia que la plataforma incide de manera positiva ya que facilita el intercambio de documentos entre el docente y estudiante, además se puede canalizar información individual del estudiante, se puede verificar su avance en el aprendizaje y sobre todo se puede incrustar, vincular herramientas interactivas que permiten un encuentro sincrónico o asincrónico más dinámico y versátil.

Los resultados obtenidos reflejan que la ejecución de la plataforma Microsoft Teams incluye de manera positiva, ya que está acompañada de estrategias metodológicas y con el docente de mediados, estando de acuerdo con (Vargas-Cubero \& Villalobos-Torres, 2017), afirman que el uso de tecnología y plataformas virtuales favorecen el aprendizaje pero por si solas no marcan la diferencia. Por lo tanto, la combinación perfecta para un aprendizaje significativo es la tecnología, estrategias metodológicas y el docente. Durante la entrevista las docentes manifestaban que incorporan a sus actividades pedagógicas, metodologías activas como ABP, Proyectos, siendo esta la oportunidad plantear una metodología diferente llamada Flipped Classroom usando la plataforma Microsoft Teams. 


\section{PROPUESTA}

Como aplicar la metodología activa Flipped Classroom utilizando Microsoft Teams, la cual está fundamentada 3 fases: Antes del encuentro virtual, durante el encuentro virtual y después del encuentro virtual (Figura 2).

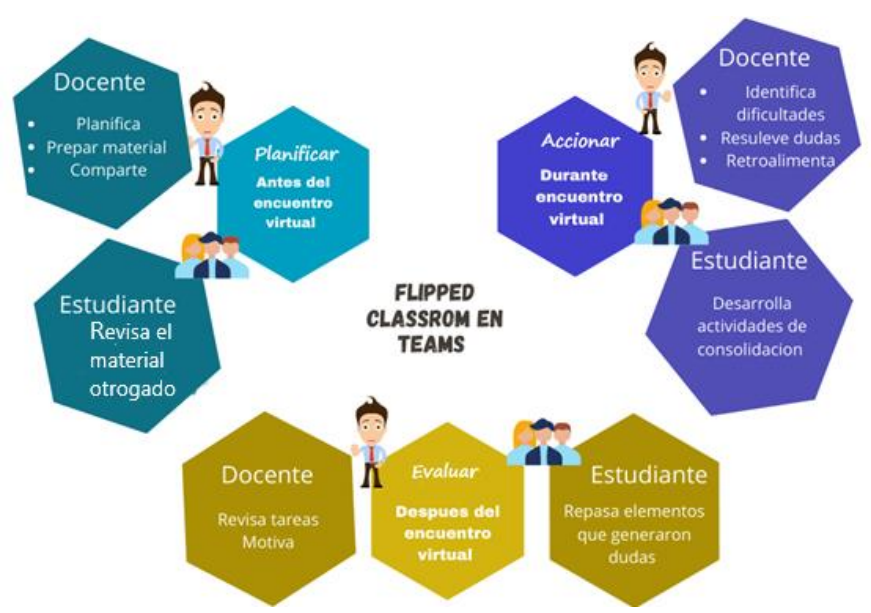

Figura 2. Esquema de propuesta Flipped Classroom en Teams. Elaboración: Los autores.

A continuación, se describe las principales fases:

Planificar: Es un proceso clave ya que es el punto de partida, en donde el docente deber examinar de forma detenida el diseño de planificación de su aula, es decir, debe estar relacionado con el currículo nacional de educación vigente, las estrategias metodológicas y los recursos digitales a emplearse. Es decir, el docente se convierte un facilitador en un entorno de retador y motivador en una modalidad de estudios virtual. Ahora bien, entre las herramientas que se pueden utilizar para la realización de las planificaciones son: Paquete de office 365 (Word, Excel, PowerPoint, SharePoint).

Preparación material: Luego de la planificación el docente debe iniciar con la creación o selección del material, herramientas que permitirá al estudiante alcanzar habilidades de orden inferior. En este punto hay dos enfoques diferentes que puede utilizar: la primera 
opción es la grabación de videos tutoriales, ya que tiene como ventaja que son más atractivos para el estudiante. Y la segunda opción es el uso archivos pdf, infografías, audios mp3.

Compartir: Es de mucha importancia que el docente analice las herramientas y recursos que va implementar durante 5 a 7 minutos y posteriormente ir asignando los videos, ejercicios de práctica, teniendo en cuenta que debe ser flexible y disponible para los estudiantes en cualquier momento.

Accionar: En este apartado el docente determinará lo aprendido mediante una lluvia de ideas utilizando la herramienta pizarra digital. Luego se formará grupos pequeños de trabajo en donde serán asignados actividades para su resolución. ¿Cómo lo hago? Pues es sencillo solo debe relacionar en el menú de la reunión en curso salas grupales y automáticamente Teams creará los grupos respectivos, el docente deberá supervisar el trabajo de los estudiantes y aplicar retroalimentación de forma personalizada 0 individualizada con el fin de obtener un aprendizaje activo. Finalizada la actividad grupal se debe reagrupar a la clase para la consolidación conceptos para lo cual podemos realizar exposiciones en PowerPoint.

Evaluar: La evaluación es parte importante ya que permite observar si es estudiante logro el aprendizaje esperado. En el caso de obtener resultados negativos el docente reprogramara actividades para que el estudiante pueda reforzar a profundidad y potenciar el aprendizaje. La evaluar se puede utilizar herramientas como Forms. 


\section{CONCLUSIONES}

Microsoft Teams como plataforma virtual pretende simular escenarios de aprendizaje como lo hacían de forma presencial, debido a que los estudiantes pueden ejecutar varias aplicaciones en un solo entorno, las mismas que le permiten organizar la información y la interacción entren compañeros de aula y docentes desarrollan competencias cognitivas. Además, se rompe el tradicionalismo con el uso de la tecnología y hace que el estudiante se empodere de su aprendizaje.

Considerando los resultados el uso de la plataforma Teams incide de manera positiva en los estudiantes, ya que es una plataforma amigable y de fácil instalación en los dispositivos electrónicos, además su manejo es sencillo por lo que no es necesario de conocimientos avanzados para su uso. Ahora bien, las docentes esta conformes con los resultados obtenidos en el proceso de enseñanza aprendizaje ya que el $84.5 \%$ acumulado en la encuesta realizada a los estudiantes dicen estar de acuerdo y totalmente de acuerdo en que Teams es de gar ayuda para el aprendizaje.

Para finalizar, es de vital importancia la mediación de profesor, ya que por sí sola una plataforma no podría alcázar los resultados esperados. Por lo que la aplicación de estrategias metodológicas y la motivación son de gran importancia para potenciar el interés por aprender de los estudiantes y por ende desarrollar habilidades y competencias tecnológicas y cognitivas para el buen desenvolvimiento en la vida diaria.

\section{FINANCIAMIENTO}

No monetario.

\section{AGRADECIMIENTO}

A la Jefatura de Posgrados de la Universidad Católica de Cuenca por permitir el desarrollo y fomento de la investigación. 
Revista Arbitrada Interdisciplinaria KOINONIA

Año VI. Vol VI. N³. Edición Especial: Educación II. 2021

Hecho el depósito de Ley: FA2016000010

ISSN: $2542-3088$

FUNDACIÓN KOINONIA (F.K). Santa Ana de Coro. Venezuela.

Cecilia Maricela Rodríguez-Guijarro; Ana Zulema Castro-Salazar

\section{REFERENCIAS CONSULTADAS}

Haro-Calero, R, \& Yépez-Pullopaxi, G. (2020). El uso de herramientas de office 365 en el proceso de enseñanza del idioma inglés. Propuesta de manual [The usage of office 365 tools in the process of English language teaching. Manual proposal]. Revista Universidad y Sociedad, 12(5), 525-530.

Hernández, R. M., Orrego Cumpa, R., \& Quiñones Rodríguez, S. (2018). Nuevas formas de aprender: La formación docente frente al uso de las tic [New ways of learning: Teacher training versus the use of TIC ]. Propósitos y Representaciones, 6(2), 671701. http://dx.doi.org/10.20511/pyr2018.v6n2.248

Lagunes-Domínguez, A. L., \& Lagunes-Domínguez, P. L. (2018). Plataformas educativas para mejorar el proceso de aprendizaje en organizaciones educativas [Educational platforms to improve the learning process in educational organizations]. Estrategias Organizacionales e Innovación Tecnológica. https://n9.cl/wvnvs

Llesquen-Chinga, R. G. (2020). Implementación de la plataforma virtual Microsoft Teams en la gestión deucativa de la institucion educativa Privada Nuestro Maravilloso Mundo,Lima, 2020 [Implementation of the virtual platform Microsoft Teams in the educational management of the Private educational institution Nuestro Maravilloso Mundo, Lima, 2020]. https://hdl.handle.net/20.500.12692/49739

Mendoza-Noriega, V. J., García-Herrera, D. G., Guevara-Vizcaíno, C. F., \& ErazoÁlvarez, J. C. (2020). Microsoft Teams como entorno virtual de la enseñanza y aprendizaje de la asignatura de Física [Microsoft Teams as a virtual environment for the teaching and learning of the Physics subject]. Cienciamatria, 6(3), 354-381. https://doi.org/10.35381/cm.v6i3.405

Pando, V. (2018). Tendencias didácticas de la educación virtual: Un enfoque interpretativo [Didactic trends in virtual education: An interpretive approach]. Propósitos y Representaciones, 6(1), 463505. https://dx.doi.org/10.20511/pyr2018.v6n1.167

Rivera-Villalta, P., García-Herrera, D., Erazo-Álvarez, J., \& Narváez-Zurita, C. (2020). Formación de competencias tecnológicas en el uso de Microsoft Teams en los estudiantes del bachillerato [Training of technological competencies in the use of Microsoft Teams in high school students]. CIENCIAMATRIA, 6(3), 543-559. https://doi.org/10.35381/cm.v6i3.414 
Revista Arbitrada Interdisciplinaria KOINONIA

Año VI. Vol VI. N³. Edición Especial: Educación II. 2021

Hecho el depósito de Ley: FA2016000010 ISSN: 2542-3088

FUNDACIÓN KOINONIA (F.K). Santa Ana de Coro. Venezuela.

Cecilia Maricela Rodríguez-Guijarro; Ana Zulema Castro-Salazar

Sánchez-Palacios, L. (2020). Impacto del Aula Virtual en el Proceso de Aprendizaje de los Estudiantes de Bachillerato General [Impact of the Virtual Classroom on the Learning Process of General High School Students]. Revista TecnológicaEducativa Docentes 2.0, 9(1), 75-82. https://doi.org/10.37843/rted.v9i1.105

Sánchez-Rodríguez, J. (2009). Plataformas de enseñanza virtual para entornos educativos [virtual education platforms for educational settings]. Pixel Bit. Revista de Medios y Educacion, 34, 18. https://n9.cl/vs1t

Vargas-Cubero, A. L., \& Villalobos-Torres, G. (2017). El uso de plataformas virtuales y su impacto en el proceso de aprendizaje en las asignaturas de las carreras de Criminología y Ciencias Policiales, de la Universidad Estatal a Distancia de Costa Rica [The use of virtual platforms and its impact on the learning process in the subjects of the Criminology and Police Sciences careers, of the State Distance University of Costa Rica]. Revista Electrónica Educare, 22(1), 1.

(C2021 por los autores. Este artículo es de acceso abierto y distribuido según los términos y condiciones de la licencia Creative Commons Atribución-NoComercial-Compartirlgual 4.0 Internacional (CC BY-NC-SA 4.0) (https://creativecommons.org/licenses/by-nc-sa/4.0/). 